\title{
EDITORIAL
}

\section{New Trends in Nano-Engineering}

\author{
Editor-in-Chief: Raffaele Barretta
}

With rapid developments of nanoengineering in the recent years, highperformance and multi-functional nanomaterials, exhibiting new and enhanced physical and chemical properties, are introduced with innumerable conceivable applications. The recent advances in design, synthesis and characterization techniques of nano-materials have enabled the fabrication of modern nano-electromechanical systems (NEMS). Research breakthroughs in NEMS technology over the past decade have caused an increased interest in the analysis of structures at nano-scale, stimulated by the fact that control over these exclusive properties leads to development of concepts potentially resulting in new engineering applications.

Nanomaterials, such as carbon nanotubes and graphene sheets, have also attracted emergent interest since the isolation of graphene due to their exclusive structural, chemical and electronic properties and their astonishing competences in sensing and bio-sensing, energy conversion and storage and bio-medical ap-

Received: July 12, 2018; Accepted: July 13, 2018; Published: July 16, 2018

Correspondence to: Raffaele Barretta, Department of Structures for Engineering and Architecture, University of Naples Federico II, Italy; Email: rabarret@unina.it

Citation: Barretta R. New Trends in Nano-Engineering. Chem Rep, 2018, 1(1):1-2

Copyright: (c) 2018 Raffaele Barretta. This is an open access article distributed under the terms of the Creative Commons Attribution License, which permits unrestricted use, distribution, and reproduction in any medium, provided the original author and source are credited. plications. Nanomaterials can be also efficiently exploited as excellent components for reinforcement in nano-composites.

It is well established that nanostructures have discrete nature manifested in the form of atoms and interactions between atoms so that classical continuum mechanics can have limited success in describing size effects. The research community of engineering science has been vigorously trying to capture the size-dependent behaviour employing nonlocal elasticity theories, among other generalized continuum mechanics approaches.

Chemical Reports is an international research journal and invites contributions of original and new fundamental research. The journal aims at providing an international forum for the presentation of highquality and rigorous research, interpretative reviews and discussion of original developments in chemical and mechanical engineering. Papers describing innovative theoretical proposals and new numerical methodologies and applications in nanoengineering are most welcome, such as those which demonstrate the transfer of techniques from other disciplines. Reports of carefully executed experiments, which are soundly interpreted, are also welcome.

The main aim of Chemical Reports is to generate bridges between the most innova- 
tive scientific developments and their potential applications in the fields of chemical and mechanical engineering applicable to nano-materials and nano-structures.

Potential topics of interest include but are not limited to the following:

- Chemical engineering

- Chemical technology

- Biochemical engineering and technology
- Green Chemistry

- Chemicals synthesis and process

- Chemicals structure

- Thermodynamics

- Reaction and catalysis

- Nanotechnology

- Chemical optimization

- Mechanics of nanostructures

- NEMS problems

- Nonlocal elasticity 